\title{
Identification of novel key amino acids at the interface of the transmembrane domains of human BST-2 and HIV-1 Vpu
}

Xiaojing Pang ${ }^{1 \dagger}$, Siqi $\mathrm{Hu}^{1+}$, Jian $\mathrm{Li}^{1}$, Fengwen $\mathrm{Xu}^{1}$, Shan Mei ${ }^{1}$, Jinming $\mathrm{Zhou}^{2}$, Shan Cen², Qi Jin ${ }^{1 *}$ and Fei Guo ${ }^{1 *}$

\begin{abstract}
Background: BST-2 (bone marrow stromal cell antigen 2) is an interferon-inducible protein that inhibits virus release by tethering viral particles to the cell surface. This antiviral activity of BST-2 is antagonized by HIV-1 accessory protein Vpu. Vpu physically interacts with BST-2 through their mutual transmembrane (TM) domains. In this study, we utilized the BRET assay and molecular dynamics (MD) simulation method to further characterize the interaction of BST-2 and Vpu.

Results: Amino acids 134, L37, P40 and L41 in the TM domain of BST-2, and L11, A18 and W22 in the TM domain of Vpu were identified to be critical for the interaction between BST-2 and Vpu. The residues P40 in the TM domain of BST-2 and L11 in the TM domain of Vpu were shown, for the first time, to be important for their interaction. Furthermore, triple-amino-acid substitutions, 14-16 (All to VAA) and 26-28 (IIE to AAA) in Vpu TM, not the single-residue mutation, profoundly disrupted BST-2Npu interaction. The results of MD simulation revealed significant conformational changes of the BST-2Npu complex as a result of mutating P40 of BST-2 and L11, 14-16 (All to VAA) and 26-28 (IIE to AAA) of Vpu. In addition, disrupting the interaction between BST-2 and Vpu rendered BST-2 resistant to Vpu antagonization.
\end{abstract}

Conclusions: Through use of the BRET assay, we identified novel key residues P40 in the TM domain of BST-2 and L11 in the TM domain of Vpu that are important for their interaction. These results add new insights into the molecular mechanism behind BST-2 antagonization by HIV-1 Vpu.

Keywords: Human immunodeficiency virus type 1, Vpu, BST-2, Bioluminescence resonance energy transfer assay, Molecular dynamics simulation

\section{Background}

The bone marrow stromal antigen 2 (BST-2, also referred to as Tetherin, CD317 or HM1.24) blocks the release of human immunodeficiency virus type 1 (HIV-1) by directly tethering viral particles to the membrane of infected cells [1,2]. BST-2 is a type II integral membrane glycoprotein of unusual topology. It comprises a short N-terminal cytoplasmic tail (CT), followed by a singlepass transmembrane domain (TM), a disulfide-linked coiled-coil ectodomain (EC), and a C-terminal glycosylphosphatidylinositol (GPI) component that acts as second

\footnotetext{
*Correspondence: zdsys@vip.sina.com; guoafei@hotmail.com ${ }^{\dagger}$ Equal contributors

'MOH Laboratory of Systems Biology of Pathogens, Institute of Pathogen Biology, Beijing 100730, P. R. China

Full list of author information is available at the end of the article
}

membrane anchor $[3,4]$. This unique membrane topology suggests that BST-2 directly tethers budding virions to the membrane of infected cells. Indeed, results of electron microscopy revealed that BST-2 is located between virus and cell membranes as well as between tethered virions [2,5-7]. Furthermore, BST-2 diminishes the infectivity of released HIV-1 particles [8]. BST-2 has also been shown to restrict viral release of other enveloped viruses, including other retroviruses, filoviruses, arenaviruses, orthomyxoviruses, gamma-herpesviruses and rhabdoviruses [9-13]. These results suggest that BST-2 is a general cellular restriction factor for enveloped viruses.

HIV-1 viral protein U (Vpu) serves as a BST-2 antagonist [2]. Vpu is an 81-amino acid, oligomeric type I trans-membrane protein encoded by an alternative open reading frame in the env gene [14]. Vpu mediates the

\section{Biomed Central}


removal of BST-2 from its site of action on the cell surface, although the exact mechanism by which Vpu affects the internalization, recycling, membrane transport, or degradation of BST-2 requires further study [4]. Phosphorylation of a pair of conserved serine residues (S52 and S56) in the cytoplasmic tail of $\mathrm{Vpu}$ is required for efficient Vpu-mediated degradation of BST-2 [15]. These phosphorylated serines residues are recognized by an Fbox-containing ubiquitin ligase subunit, $\beta$-TrCP-2. Vpu thus recruits the multisubunit SCF- $\beta-\operatorname{TrCP}$ E3 ubiquitin ligase complex that causes ubiquitination and degradation of BST-2 [15-18]. Binding of Vpu to BST-2 through their transmembrane domains is crucial for the antagonization [16,19-22]. NMR studies have shown that BST-2 and Vpu directly contact between their transmembrane domains [16]. Studies have revealed that mutation in the transmembrane of either BST-2 (L22, L23, G25, I26, V30, I33, I34, I36, L37, L41, and T45) or Vpu (A14, A18 and W22) renders BST-2 resistant to Vpu [16,23-25].

Bioluminescence resonance energy transfer (BRET) assay has been used for real-time monitoring of proteinprotein interactions in live cells [26]. The nonradiative (dipole-dipole) transfer of energy from donor enzyme to complementary acceptor fluorophore occurs after substrate oxidation. Donor to acceptor energy transfer and consequent emission from acceptor generally indicates less than $10 \mathrm{~nm}$ of separation of the two proteins, suggesting that the protein of interest are likely to be interacting with each other (directly or as part of a complex) [27]. Disruption or deficiency of interaction between donor and acceptor proteins results in decreased donor to acceptor energy transfer. BRET has been used to study a wide range of protein-protein interactions in bacterial, plant and mammalian cells [28-30]. This technique has been previously utilized to study the possible effects of small molecules on BST-2/Vpu interaction [31].

In this study, we utilized the BRET assay to identify the amino acids in the TM regions of human BST-2 and HIV-1 Vpu that are required for their interaction in live cells. Several amino acids on each transmembrane domain were found crucial for the interaction of BST-2 and Vpu (BST-2: I34, L37, P40 and L41; Vpu: L11, A18 and W22). In addition to some of these interaction sites that were reported in other studies $[24,25]$, our data further showed that P40 in the TM domain of BST-2 and L11 in the TM domain of Vpu were also important for this interaction. Furthermore, we observed that tripleamino-acid mutants, 14-16 (AII to VAA) and 26-28 (IIE to AAA) in Vpu TM significantly affected the interaction, but not seen for single-residue mutation. The novel residues of P40A of BST-2 and L11A, 14-16 (AII to VAA) and 26-28 (IIE to AAA) of Vpu led to unstable complex compared to the wild-type (WT) as shown by the results of molecular dynamics (MD) simulation. Further studies showed that loss of interaction between the two proteins directly affected the Vpu-mediated BST-2 degradation and the inhibition of viral release.

\section{Results}

Rluc-BST-2 and Vm-EYFP are suitable for BRET assay

BRET signal was generated using Rluc (Renilla luciferase) and EYFP (Enhanced Yellow Fluorescent Protein) in this study. Rluc or EYFP was fused with human BST-2 or HIV-1 Vpu, respectively. In order to rule out the effect of BST-2 degradation by Vpu, the S52, 56A mutation of Vpu was used in the BRET study (Figure 1a). This is a degradation-null mutant but still interacts with BST-2, and is hereafter referred to as Vm. Eight plasmids were generated, namely Rluc-BST2 (R-B), EYFP-BST2 (E-B), BST2-Rluc (B-R), BST2-EYFP(B-E), Rluc-Vm(R$V m)$, EYFP-Vm(E-Vm), Vm-Rluc (Vm-R), Vm-EYFP(VmE) (Figure 1b). Most of the fusion proteins were expressed well in transfected HEK 293 T cells except for B-E, B-R and Vm-R that had lower expression levels (Figure 1c). Every pair of fusion proteins, containing BST-2 and Vm fused with Rluc or EYFP, were cotransfected into HEK293T cells. R-B and Vm-E produced BRET signal as strong as the positive control R-E (Rluc fused with EYFP directly) (Figure 1d). We therefore used the R-B/Vm-E pair in the subsequent BRET assay to study BST-2 and Vpu interaction (Figure 1e). We next investigated the subcellular localization of the wild-type and mutated proteins. The results in Figure $1 \mathrm{f}$ revealed that R-B and Vm-E had localization similar to wild-type proteins. R-B and Vm-E were expressed at levels similar to the untagged, wild-type BST-2 and Vpu. HIV-1 VLP release was inhibited by R-B. In addition, V$E$ induced the degradation of R-B, and enhanced HIV-1 VLP release, while Vm-E neither degraded R-B nor rescued HIV-1 VLP release (Figure 1g).

\section{Use BRET assay to monitor the interaction of BST-2 and Vpu in live cells}

In order to determine the specificity of the interaction between BST-2 and Vpu, we substituted Vm with unrelated protein CD209 which is a type II transmembrane protein consisting of $\mathrm{C}$-terminal extracellular domain, transmembrane region and $\mathrm{N}$-terminal cytoplasmic region to perform BRET assay. The BRET signal was as low as the negative control R/E (Figure 2a). We further performed saturation and competition studies to validate the specificity of the BRET assay [26]. HEK293T cells were co-transfected with fixed amounts of plasmid expressing Rluc fusion protein R-B and increasing amounts of plasmid expressing EYFP fusion protein Vm-E. The saturation curve was generated, which showed that the 
b

a

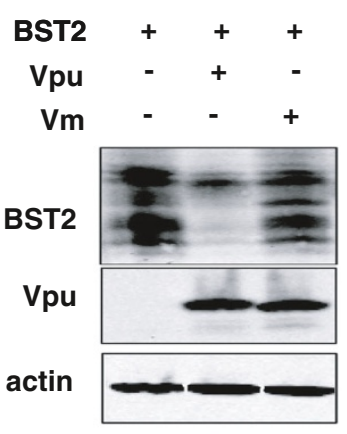

C R-B B-R B-E E-B

BST2

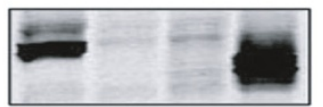

Vm-R R-Vm Vm-E E-Vm

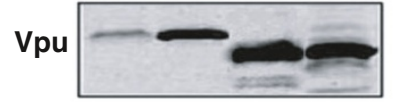

d

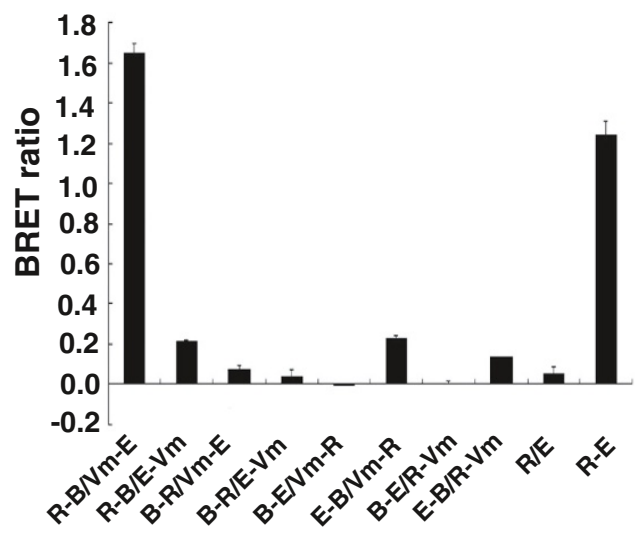

f

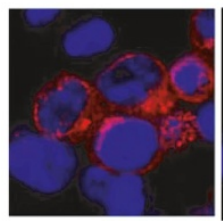

BST2

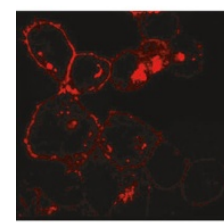

R-B
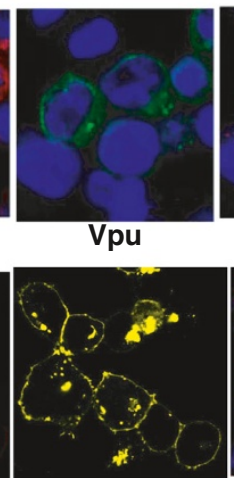

Vm-E

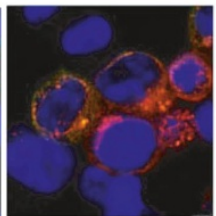

merge

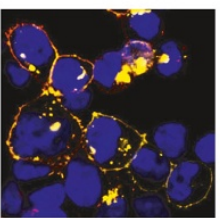

merge

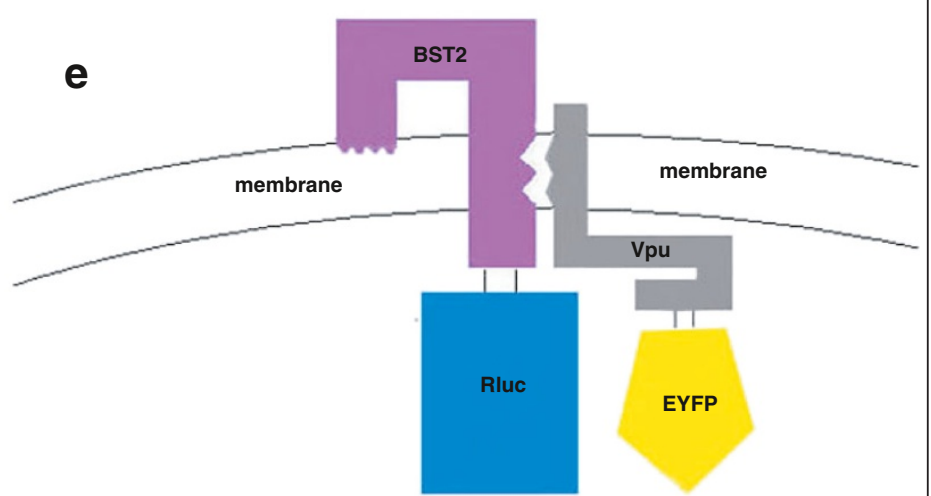

BRET

\section{g}
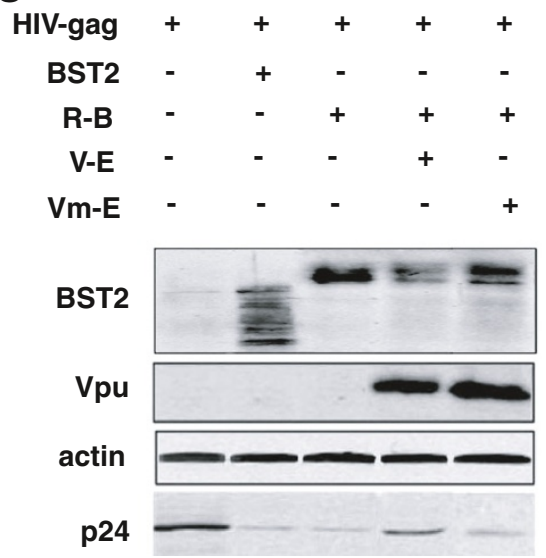

Figure 1 (See legend on next page.) 
(See figure on previous page.)

Figure 1 BRET assay to monitor BST-2/Vpu interaction. (a) Western blot analysis of cellular lysates following the cotransfection of BST-2 and Vpu or Vm plasmids into HEK 293 T cells. (b) Schematic representation of the plasmid DNA constructs. R-B and E-B were constructed with either Rluc or EYFP fused to the N terminus of BST-2; B-R and B-E were constructed with either Rluc or EYFP fused to the C terminus of BST-2; The $\mathrm{N}$-terminus of Vm was fused with Rluc and EYFP to generate R-Vm and E-Vm; The C-terminus of Vpu was fused with Rluc and EYFP to engineer $V m-R$ and $V m-E$. (c) Expression of the fusion proteins. Western blot analysis of the cellular lysates following the transfection of the expressing plasmid into HEK 293 T cells using anti-BST-2 and Vpu antibodies. (d) The BRET signals of BST-2 and Vpu interaction. HEK293T cells were transfected with different combinations of donor and acceptor DNA constructs. The BRET ratio was measured using a Pekin Elmer reader followed by the addition of substrate Coelenterazine at the final concentration of $5 \mu \mathrm{M}$. (e) The schematic diagram of the energy transfer between R-B and V-E. (f) Subcellular localization of R-B and Vm-E. Immunofluorescence staining was performed to detect the localization of BST-2 (red), Vpu (green), R-B(red) and Vm-E(yellow) expressed in HEK 293 T cells. The nuclei were stained with DAPI (blue). Representative images are shown. (g) Function analysis of the tagged BST-2 and Vpu proteins. Western blot analysis of the cellular lysates and the corresponding viral lysates following cotransfection of the HIV-1 Gag-Pol construct (containing gag and pol genes) and other expressing plasmids into HEK293T cells using anti-BST-2, anti-Vpu, anti-actin and anti-p24 antibodies.

BRET signal reached a saturation level with high concentrations of Vm-E. This phenomenon was not observed in cells co-expressing E-CD209 protein and R-B (Figure 2b). In addition, constant amounts of R-B and $\mathrm{Vm}-\mathrm{E}$ proteins were co-expressed with untagged $\mathrm{Vm}$ that competed for interaction with the tagged protein Vm-E. A reduction of the BRET ratio was observed.
Increasing the amounts of untagged $\mathrm{Vm}$ diminished the BRET ratio in a dose-dependent manner. Again, this phenomenon was not observed in cells co-expressing untagged protein CD209 that did not interact specifically with R-B (Figure 2c). These results indicate that the BRET assay is a useful and sensitive approach to study BST-2 and Vpu interaction in live cells.

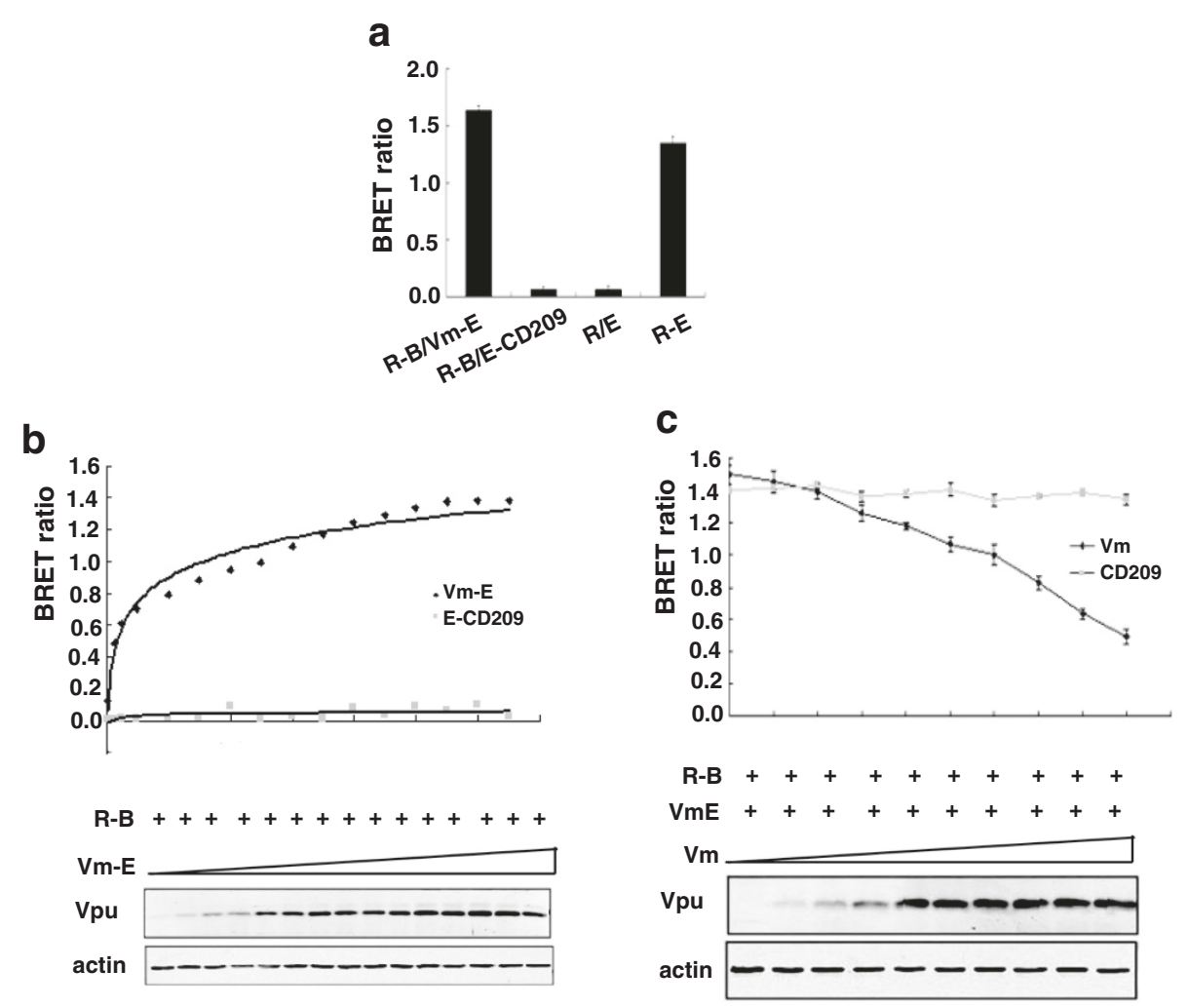

Figure 2 The specificity of the BRET assay in detecting the interaction of BST-2 and HIV-1 Vpu. (a) The specificity of BRET signals between BST-2 and Vpu. HEK293T cells were transfected with plasmid DNA and seeded into 96-well plates, and then the BRET ratio was measured by using a Pekin Elmer reader. (b) The saturation curve of the BRET assay. HEK293T cells were cotransfected with constant amount of R-B plasmid and increasing dose of Vm-E or E-CD209 plasmid. The BRET ratio was detected by using a Pekin Elmer reader. Western blot analysis of the cellular lysates was shown. (c) The competition curve of the BRET assay. HEK293T cells were cotransfected with constant amount of R-B plasmid and Vm-E and increasing dose of Vm or CD209 plasmid, and the BRET ratio was detected by using a Pekin Elmer reader. Western blot analysis of the cellular lysates was shown. 


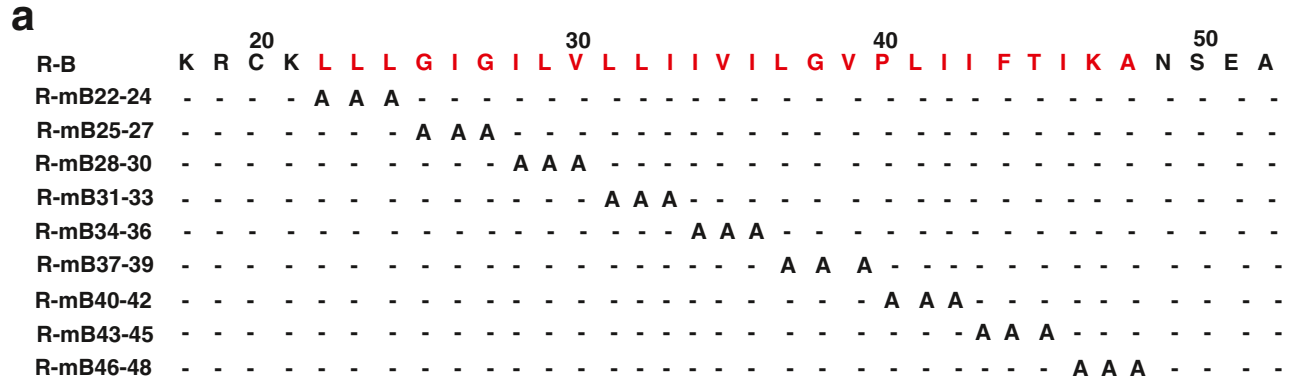

b

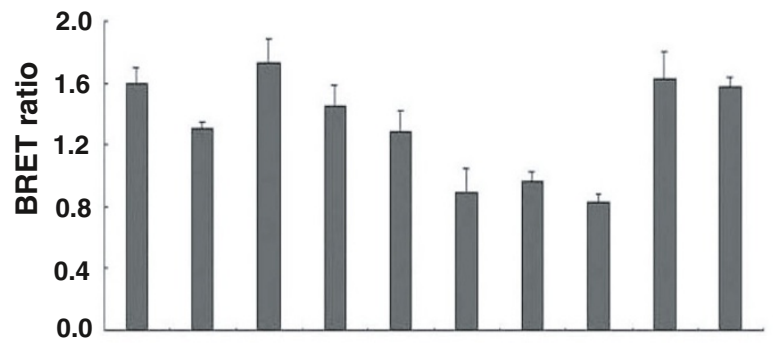

BST2

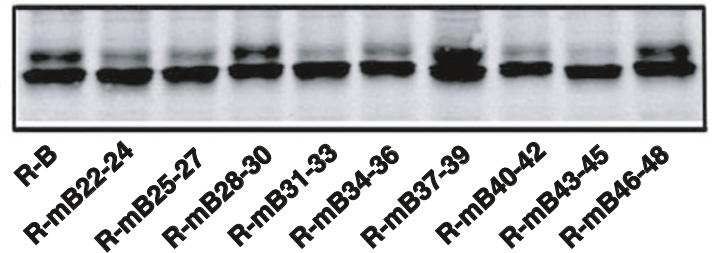

C

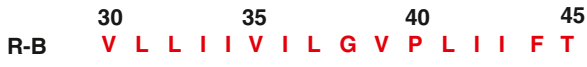

R-mB34 - - - - A - - - - - - - - - - -

R-mB35 - - - - - A - - - - - - - - - -

R-mB36 - - - - - A - - - - - - - -

R-mB37 - - - - - - A - - - - - - - -

R-mB38 - - - - - - - A - - - - - -

R-mB39 - - - - - - - A - - - - -

R-mB40 - - - - - - - - - A - - - -

R-mB41 - - - - - - - - - - - A - - - -

R-mB42 - - - - - - - - - - A - -

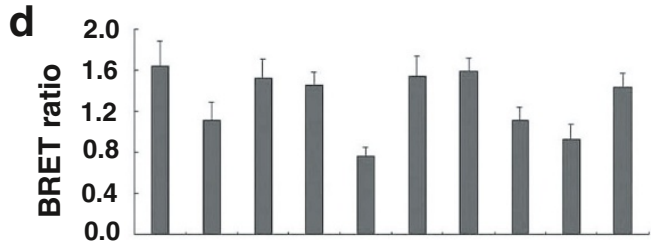

BST2

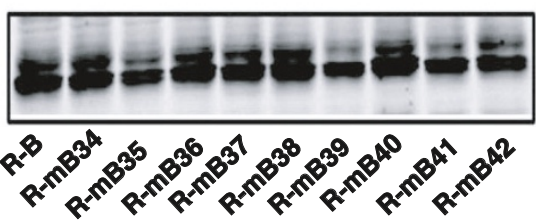

Figure $\mathbf{3}$ (See legend on next page.) 
(See figure on previous page.)

Figure 3 The amino acids in TMD of human BST-2 responsible for HIV-1 Vpu interaction. (a) Mutation of the R-B TM domain. Every three amino acids are mutated as a group. (b) HEK293T cells were cotransfected with Vm-E plasmid and R-B TM mutants, and the BRET ratio was detected by using a Pekin Elmer reader. Western blot analysis of the cellular lysates was shown. (c) Mutation of R-B TM domain. (d) HEK293T cells were cotransfected with Vm-E plasmid and a series of the R-B TM single amino acid mutants, and the BRET ratio was detected by using a Pekin Elmer reader. Western blot analysis of the cellular lysates was shown.

\section{Identify the amino acids in the transmembrane domains of human BST-2 and HIV-1 Vpu critical for their interaction}

Previous studies have demonstrated that the interaction between human BST-2 and HIV-1 Vpu is mediated through their TM domains [20,21]. Further detailed mutagenesis studies revealed that multiple amino acid residues across the TM domains govern the interaction between human BST-2 and HIV-1 Vpu [23-25]. In this study, comprehensive mutagenesis and BRET assay were performed to further identify residues in TM domains of BST-2 and Vpu that are important for their interaction. Plasmids encoding R-B mutants with triple-alanine substitutions in TM domain of BST-2 (amino acids 22-48) were constructed. These mutants were expressed at levels comparable to the wild type (Figure $3 \mathrm{a}$ and $3 \mathrm{~b}$ ). We then transfected these mutants together with Vm-E plasmid into HEK293T cells. Forty-eight hours posttransfection, BRET assay was performed to determine the interaction between $\mathrm{R}-\mathrm{B}$ and $\mathrm{Vm}-\mathrm{E}$. The $\mathrm{R}-\mathrm{mB}_{34-36}$, $\mathrm{R}-\mathrm{mB}_{37-39}, \mathrm{R}-\mathrm{mB}_{40-42}$ mutants had significantly reduced BRET signal compared to that of wild type R-B (Figure $3 \mathrm{~b}$ ), indicating that the amino acids from position 34 to 42 were crucial for BST-2 and Vpu interaction in live cells. Nine single-alanine-substituted mu tants from position 34 to 42 were constructed to further identify the key amino acids (Figure 3c). These nine mutants were expressed at wild type levels (Figure 3d). Mutation of residues I34, L37, P40 and L41 in TM domain of BST-2 reduced BRET signals. A novel residue P40 was shown for the first time being critical for BST-2 and $\mathrm{Vpu}$ interaction.

In order to identify the residues in $\mathrm{Vpu}$ that are important for binding to BST-2, plasmids encoding Vm-E mutants with triple-amino acid substitutions in TMD of $\mathrm{Vpu}$ (amino acids 5-27) were constructed with non-alanine to alanine residues and alanine to the same hydrophobic valine residues (Figure 4a). All mutants were expressed well in transfected HEK293T cells (Figure 4b). Among the eight triple-amino acids-subs tituted mutants, $\mathrm{mVm}_{11-13}-\mathrm{E}, \mathrm{mVm}_{14-16}-\mathrm{E}, \mathrm{mVm}_{17-19}-\mathrm{E}$, $\mathrm{mVm}_{20-22}-\mathrm{E}, \mathrm{mVm}_{26-28}-\mathrm{E}$ had markedly reduced BRET signal (Figure 4b). In order to determine the specific amino acids responsible for the interaction between $\mathrm{Vpu}$ and BST-2, each amino acid in Vm-E from positions 11 to 22 and 26 to 28 was mutated with non-alanine to alanine residues and alanine to valine residues (Figure $4 \mathrm{c}$ ).
All mutants had the same expression levels compared with $\mathrm{Vm}-\mathrm{E}$ (Figure $4 \mathrm{~d}$ ). The $\mathrm{mVm}_{18}-\mathrm{E}$ and $\mathrm{mVm}_{22}-\mathrm{E}$ mutants showed significantly decreased BRET ratio, and $\mathrm{mVm}_{11}$-E mutant had decreased BRET ratio to a lesser extent (Figure 4d). Unexpectedly, some triple substitutions in the Vm TMD had significantly decreased BRET signal, such as $\mathrm{mVm}_{14-16^{-}} \mathrm{E}$ and $\mathrm{mVm}_{26-28}-\mathrm{E}$, but none of a single mutation of the three residues generated such an effect.

\section{Effect of TM mutations on BST-2/Vpu interaction by molecular dynamics simulation}

We further investigated how the mutants that were first identified in our study affect the interaction between BST2 and Vpu by performing molecular dynamics (MD) simulation. The simulation models were shown in Figure 5a. Cluster analysis was also performed to generate the representative complex structure of the largest family during the simulation. For all the mutant complexes, the conformation of Vpu-TM fits to that of the WT complex very well (Figure 5b). Thus, we aligned the representative structure of the mutant complex with the WT complex through fitting the Vpu-TM structure, and the results are shown in Figure 5c-f. Briefly, compared to the wild type (WT) complex, the mutants showed less stable complexes which were similar to previous simulation results of the complex with I34, L37, or L41 in the TM domain of BST-2, as well as A14, A18 or W22 in the TM domain of Vpu [32]. To evaluate the overall conformational change of mutant complexes, the fitting RMSD values were calculated for each mutant complex through the alignment with the WT complex. The RMSD values of P40A mutant at BST-2, L11A mutant, 14-16 (AII to VAA) mutant, or 26-28 (IIE to AAA) mutant at Vpu-TM are $1.94 \AA$, $1.52 \AA, 0.85 \AA$, or $2.25 \AA$ respectively, which means a close overall conformation comparing to the WT complex. However, a destruction of helix at 38-41 position of BST2-TM was observed for all mutants as a result of simulation. P40A mutant in BST-2 (Figure 5c) or 26-28 (IIE to AAA) mutant in Vpu (Figure 5f) cause more significant conformational change than the other two mutants. Also, L11A mutant or 14-16 (AII to VAA) mutant in Vpu-TM destabilizes the helix conformation of 23-25 motif in Vpu but reconstructs the helix structure of 26-30 motif (Figure 5d, 5e). Thus, marked local conformation changes are seen for all four mutants, and such conformational changes likely disturb the interaction between BST-2 and Vpu. 
a

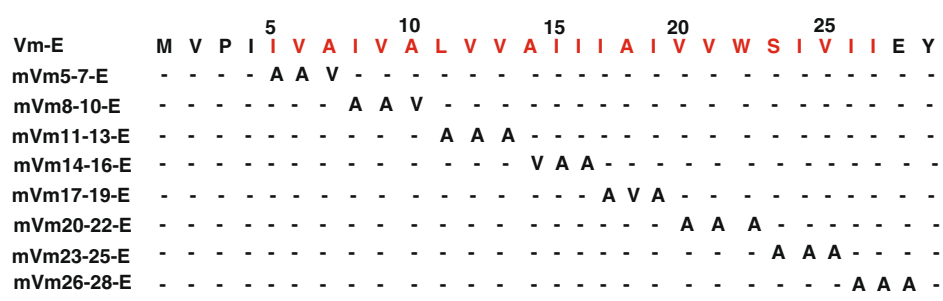

b

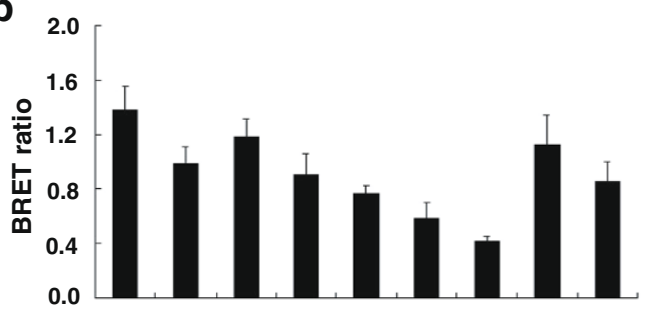

Vpu
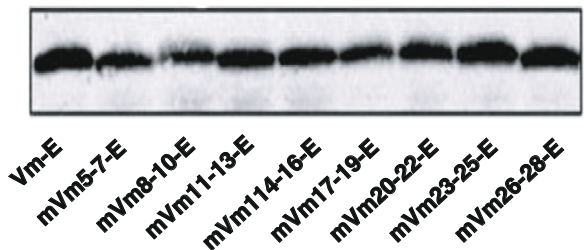

C

Vm-E $\quad \stackrel{10}{A}$ L V V V V A $\quad \begin{gathered}15 \\ \text { I I I I A I }\end{gathered}$

mVm11-E - A - - - - - - - - - - - - -

mVm12-E - - A - - - - - - - - - - . - -

mVm13-E - - - A - - - - - - - - - - - - -

mVm14-E - - - V - - - - - - - - - - -

mVm15-E - - - - - A - - - - - - - - - - - -

mVm16-E - - - - - - A - - - - - - - - - - -

mVm17-E - - - - - - A - - - - - - - -

mVm18-E - - - - - - - V - - - - - - - - -

mVm19-E - - - - - - - - - A - - - - - - - -

mVm20-E - - - - - - - - - A - - - - - - -

mVm21-E - - - - - - - - - - A - - - - - -

mVm22-E - - - - - - - - - - - A - - - - - -

mVm26-E - - - - - - - . - - - - - - - A - -

mVm27-E - - - - - - - - - - - - - - - - A - -

mVm28-E - - - . - . . . - - - - - - - - A -

d

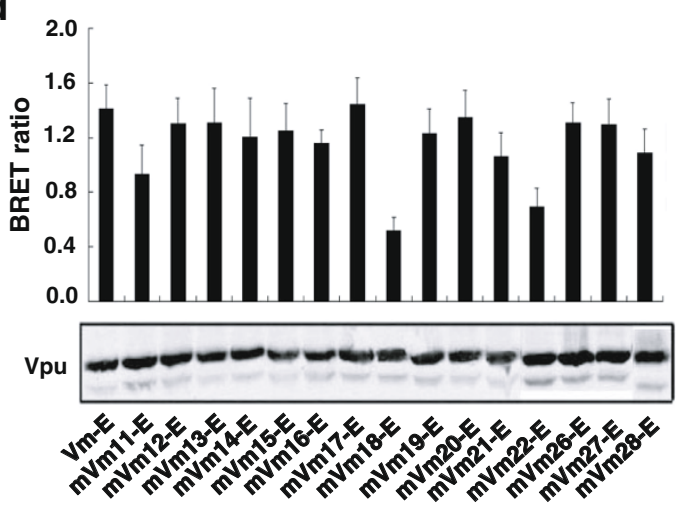

Figure 4 (See legend on next page.) 
(See figure on previous page.)

Figure 4 The amino acids in TMD of HIV-1 Vpu responsible for human BST-2 interaction. (a) Mutation of the Vm-E TM domain. Every three amino acids were mutated as a group. (b) HEK293T cells were cotransfected with R-B plasmid and a series of Vm-E TM mutants, and the BRET ratio was detected by using a Pekin Elmer reader. Western blot analysis of the cellular lysates was shown. (c) Mutation of the R-B TM domain. (d) HEK293T cells were cotransfected with R-B plasmid and a series of Vm-E TM single amino acid mutants, and the BRET ratio was detected by using a Pekin Elmer reader. Western blot analysis of the cellular lysates was shown.

Functional validation of the identified key residues in the TM domains of human BST-2 and HIV-1 Vpu

To verify the functional role of the identified residues in TM domain of BST-2, we tested these BST-2 mutants for their sensitivity to Vpu. HEK293T cells were cotransfected with HIV-1 Gag-Pol construct (containing gag and pol genes) and Vpu together with a WT R-B or $\mathrm{R}-\mathrm{B}$ mutants. The virus-like particles (VLP) in the culture supernatants together with proteins expressed in cell extract were assessed by Western blotting. As shown in Figure $6 \mathrm{a}$ and $6 \mathrm{c}$, the mutants the mutants $\mathrm{R}-\mathrm{mB}_{34}$, $\mathrm{R}-\mathrm{mB}_{37}, \mathrm{R}-\mathrm{mB}_{40}$ and $\mathrm{R}-\mathrm{mB}_{41}$ became partially or completely resistant to $\mathrm{Vpu}$ antagonization. To determine whether mutants resistant to $\mathrm{Vpu}$ antagonization are also resistant to the degradation by Vpu, BST-2 in cellular lysates was examined by Western blotting. The results showed that $\mathrm{R}-\mathrm{mB}_{34}, \mathrm{R}-\mathrm{mB}_{37}, \mathrm{R}-\mathrm{mB}_{40}$ and $\mathrm{R}-\mathrm{mB}_{41}$ were not degraded by $\mathrm{Vpu}$ as much as wild type BST-2 (Figure $6 \mathrm{a}, 6 \mathrm{~b}$ ). In order to rule out the possible effect of the Rluc sequence on the ability of BST-2 to inhibit virus

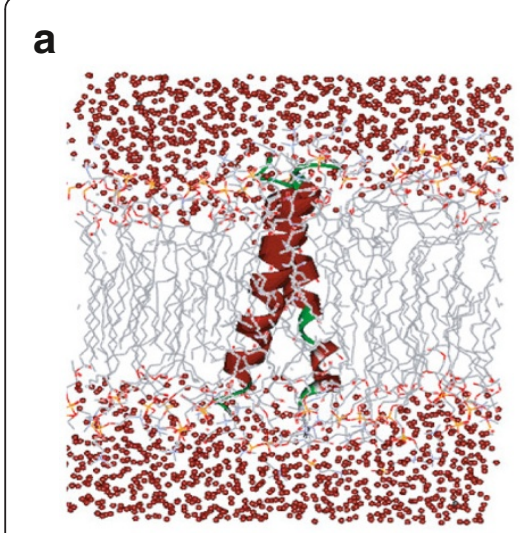

d

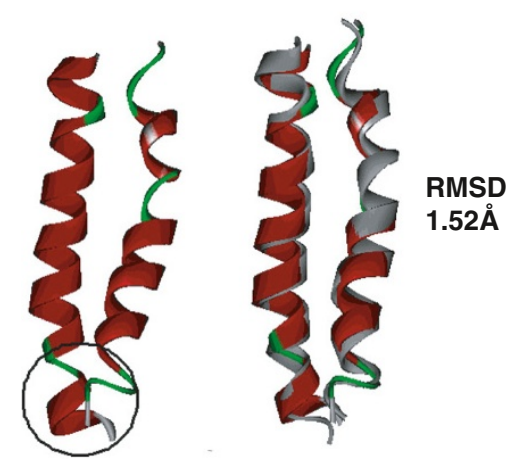

Vpu L11A b

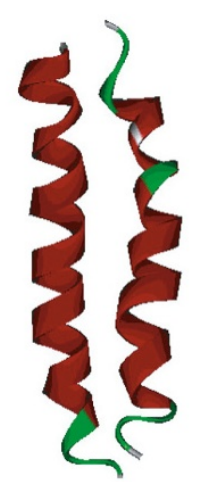

WT
C

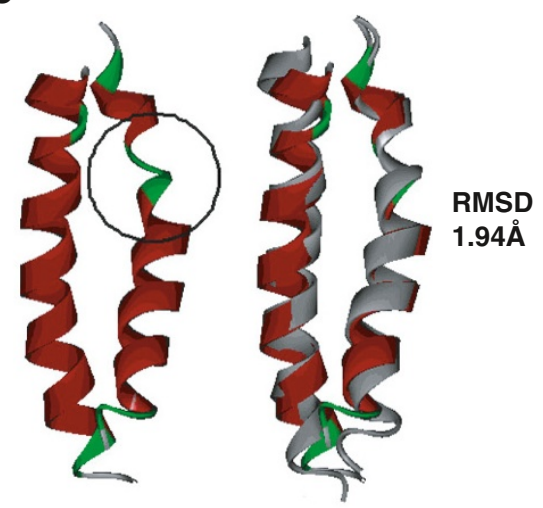

BST P40A e

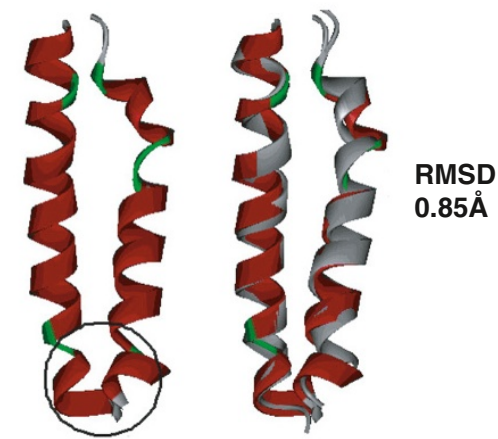

Vpu 14-16(All-VAA) f

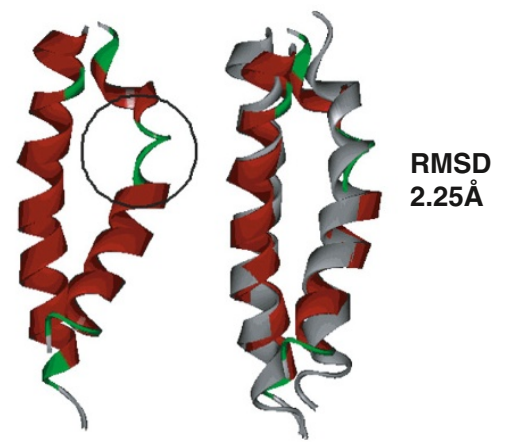

Vpu 26-28 (IIE-AAA)

Figure 5 Structures of BST-2/Vpu complex determined by molecular dynamics simulations. The representative structure was generated by cluster analysis, and the alignment of the mutant complexes (in ribbon, colored by secondary structure, helixes are in red, and turns or loops are in green) with the wild-type complex (gray ribbons) was conducted in DS (c-f). The significant conformational changes between the WT and mutants are highlighted by the black circles. (a) Description of the simulation model in membrane; (b) Representative structures of the complex of the wild type (WT) BST-TM and Vpu-TM; (c) Effect of the P40A mutation in BST2-TM on the BST-2 - Vpu interaction. (d) Effect of the L11A mutation in Vpu-TM on the BST-2 - Vpu interaction. (e) Effect of the 14-16 (All to VAA) mutation in Vpu-TM on the BST-2 - Vpu interaction. (f) Effect of the 26-28 (IIE to AAA) mutation in Vpu-TM on the BST-2 - Vpu interaction. 

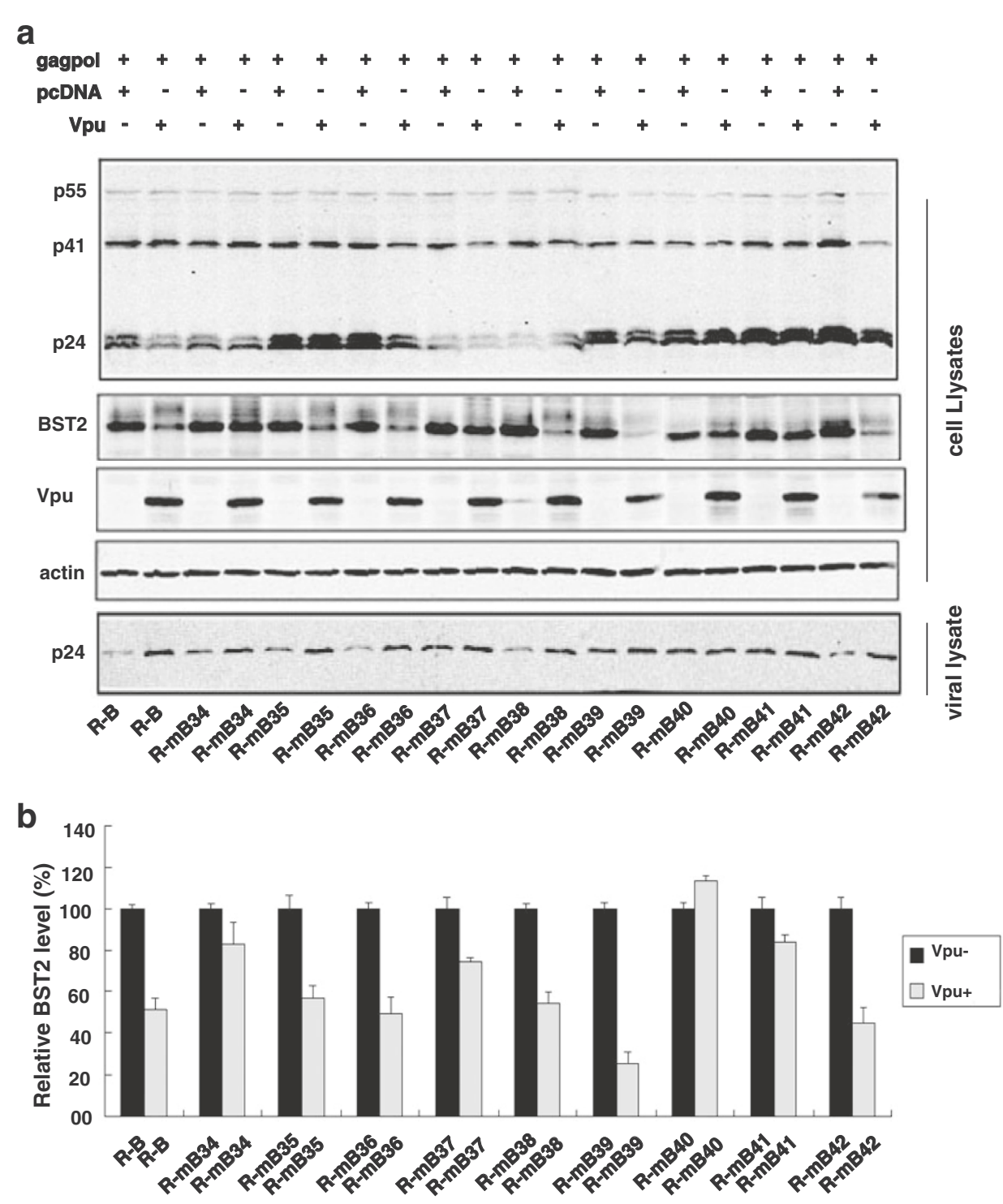

C

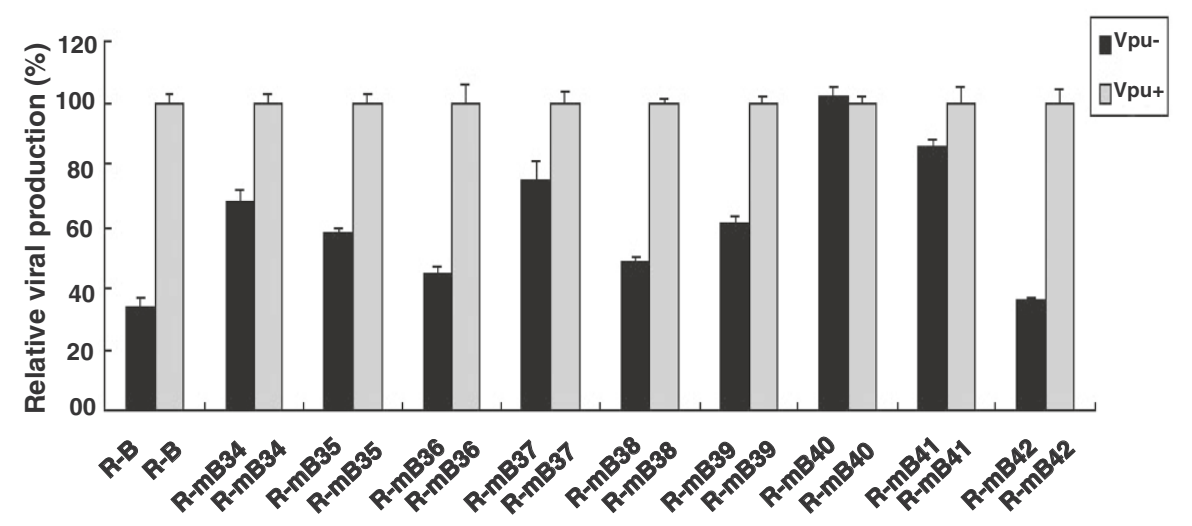

Figure 6 (See legend on next page.) 
(See figure on previous page.)

Figure 6 Functional validation of the crucial residues in the TMD of BST-2 for Vpu interaction. (a) Western blot analysis of the cellular lysates and the corresponding viral lysates following the cotransfection of the HIV-1 Gag-Pol construct (containing gag and pol genes) and other plasmids into HEK293T cells. (b) Relative BST-2 reduction was determined by densitometric scanning using the ImageJ program (NIH) aiming at the specific bands. BST-2 level in cells that were cotransfected with R-B or R-B mutants and together with pcDNA was arbitrarily set as $100 \%$. The data shown was the average of three independent experiments. (c) Relative HIV-1 VLPs release was assessed by densitometric scanning using the ImageJ program (NIH). The level of HIV-1 VLPs released from cells that were cotransfected of the HIV-1 Gag-Pol construct and R-B or R-B mutants and together with pcDNA was arbitrarily set as 100\%. The data shown was the average of three independent experiments.

release, we also inserted the above mutations into the wild type BST-2 and observed similar results (Additional file 1: Figure S1a). These data provided direct evidence that I34, L37, P40 and L41 of human BST-2 determine $\mathrm{Vpu}$ susceptibility in addition to Vpu interaction. The loss of interaction between the two proteins directly affected the subsequent BST-2 degradation and the inhibition of viral release (Figure 6a, 6c).

To further characterize the function of the residues in Vpu TMD, a series of Vpu TM crucial mutants were constructed containing the wild type S52 and S56 residues. HEK293T cells were cotransfected with HIV-1 Gag-Pol construct and BST-2 together with a series of key amino acid substituted V-E TM mutants. The VLP in the culture supernatants together with proteins expressed in cell extract were determined by Western blotting. The results showed that mutants $\mathrm{mV}_{11}-\mathrm{E}$, $\mathrm{mV}_{18}-\mathrm{E}, \mathrm{mV}_{22}$-E partially lost their ability to promote HIV-1 VLP release (Figure 7a, 7c). In the meantime, $\mathrm{mV}_{11}$-E, $\mathrm{mV}_{18}-\mathrm{E}, \mathrm{mV}_{22}$-E were unable to degrade BST-2 (Figure $7 \mathrm{a}, 7 \mathrm{~b}$ ). We also tested the above Vpu mutations by inserting them into the wild type $\mathrm{Vpu}$ and observed similar results (Additional file 1: Figure S1b). These results indicated that the L11, A18 and W22 residues in Vpu TM domain are essential for Vpu to counteract human BST-2 (Figure 7a, 7c).

\section{Discussion}

BRET signal is a ratio of the EYFP long wave emission $(530 \mathrm{~nm})$ divided by the Rluc short wave emission $(475 \mathrm{~nm})$ [27]. The BRET based assay was for the first time used by Kuhl et al. to monitor BST-2/Vpu interaction with the aim to study the effect of small molecules in this regard [31]. A similar flow cytometry-based FRET assay was used by Schildler et al. to identify novel protein interaction partners in a high-throughput format [33]. Here we used BRET assay to identify the crucial amino acids in TM of the two proteins. In our study, Vm (S52, 56A mutant) were utilized instead of wild type Vpu because Vpu degrades BST-2 and this will complicate the study of Vpu/BST-2 interaction. After testing different combinations of fusion proteins in BRET assay, we selected the group of BST-2 fused with Rluc at $\mathrm{N}$-terminal and Vm fused with EYFP at C-terminal. Previous studies showed that tagging human BST-2 at N- terminus and tagging $\mathrm{Vpu}$ at $\mathrm{C}$-terminus do not interfere with their function and cellular localization [24].

This technique allowed us to successfully identify four amino acid residues in human BST-2 TM (I34, L37, P40 and L41) and three amino acid residues in Vpu TM (L11, A18 and W22) that are required for their interaction. Findings with I34, L37 and L41 in BST-2 TM and A14, A18 and W22 in Vpu TM were reported by other groups $[24,25]$. However, the other two sites, P40 in BST-2 TM and L11 in Vpu TM were shown for the first time in this study as the critical determinants for $\mathrm{Vpu} / \mathrm{BST}-2$ interaction. It was reported that P40A mutation abolished susceptibility to $\mathrm{Vpu}$ without affecting the interaction of BST-2 and Vpu [24]. In contrast, our results showed that the P40A mutation affected this interaction to the same degree as I34A in our BRET assay. This discrepancy was likely due to the different approaches that were used to characterize BST-2 and Vpu interaction. As opposed to the BRET assay that we used, the BiFC method was employed in their study to monitor BST2 and Vpu interaction. In addition, a recent study based on cyteine cross-linking support the interaction between BST-2 P40C and Vpu A7C [34]. In addition, we replaced alanine in $\mathrm{Vpu} \mathrm{TM}$ with valine and did not observe the effects of A14L and A14F as previously reported, but the A18V effect is in line with that shown for A18L and A18F [25,35]. However, the triple-amino-acids substitution of 14-16 (AII to VAA) produced significantly decreased BRET ratio. The same result was also seen in the triple-amino-acids substitution of 26-28 (IIE to AAA). Namely, substitution of multiple amino acids, but not a single residue mutation, significantly affects the interaction. It was reported that deletion of residues I15, I16 and I17 or deletion of I26 and I27 in Vpu TM potently block the physical interaction between human BST-2 and Vpu, and prevent $\mathrm{Vpu}$ from enhancing HIV-1 virus release [36]. These results indicated that multiple isoleucine in HIV-1 Vpu TM play an important role in forming the hydrophobic interaction surface between the two proteins. Different from other reports, we observed that L11 in Vpu TM also contributes to the interaction between the two proteins albeit to a lesser extent.

In summary, results of our BRET assays identified novel residues in the TM domains in human BST-2 and 


\section{a}
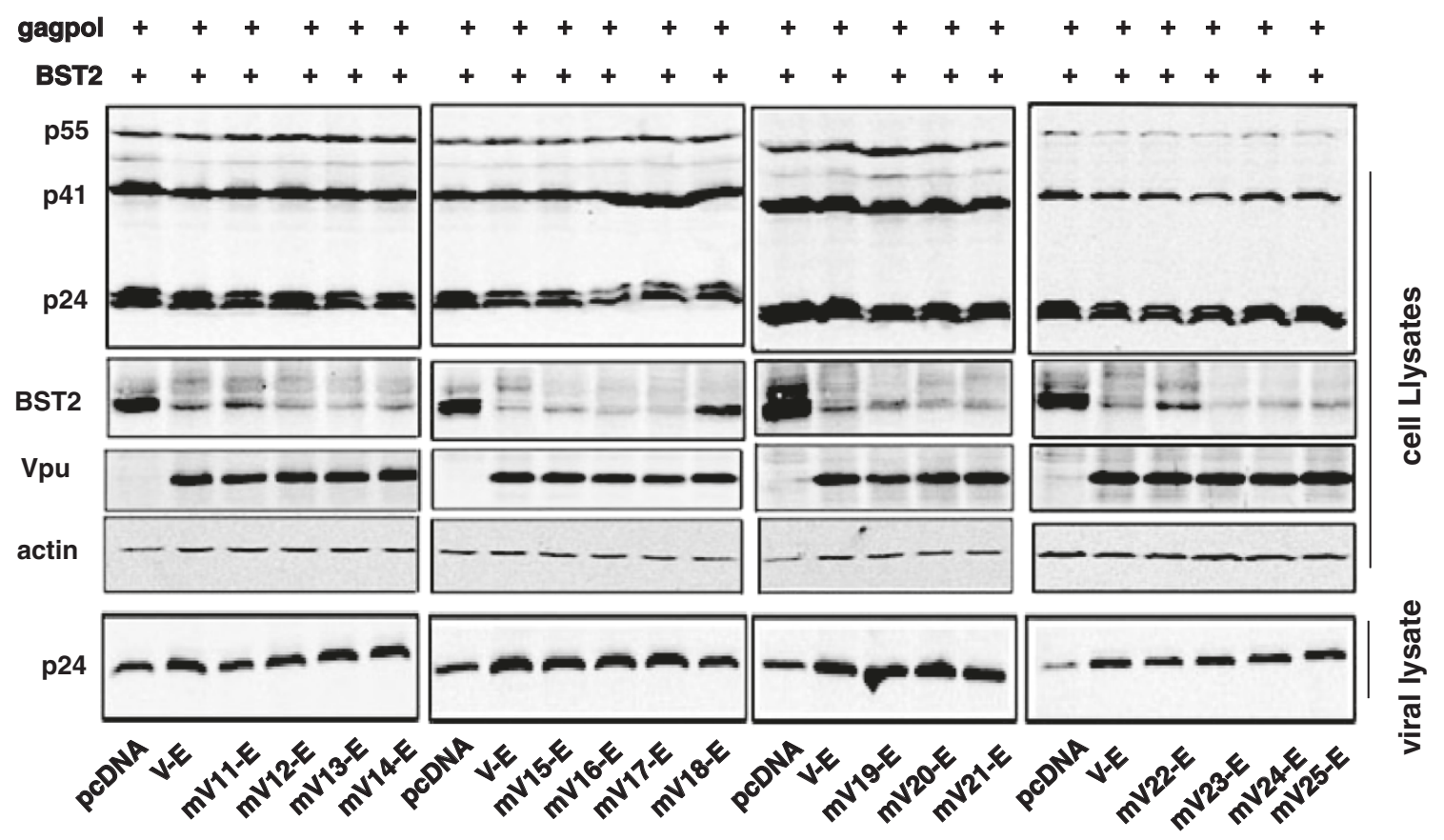

b

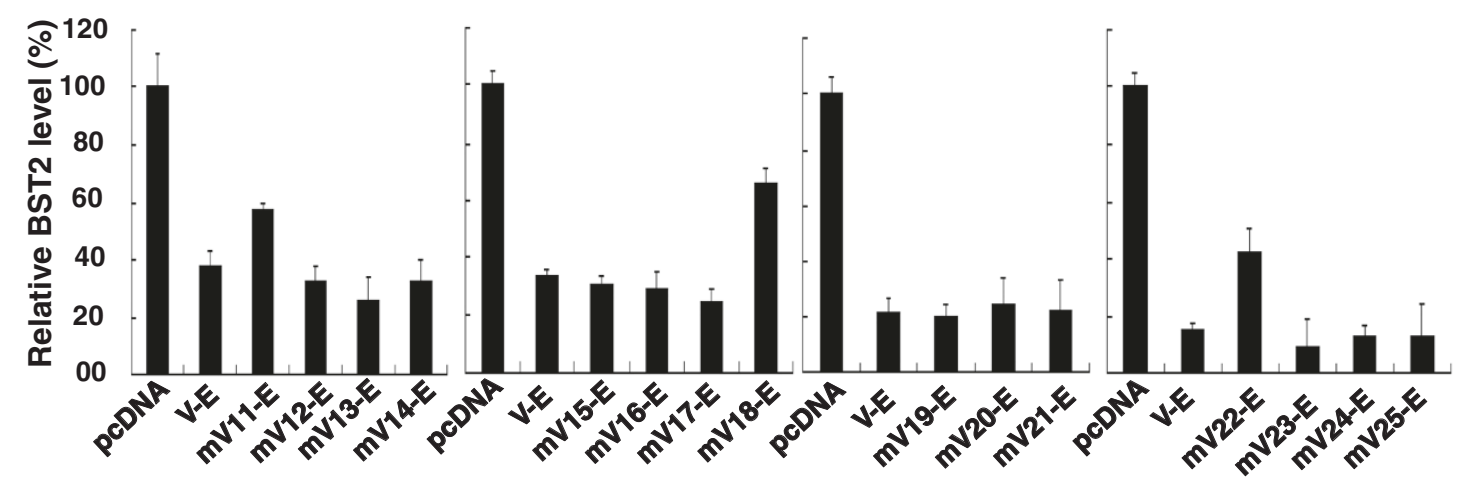

C

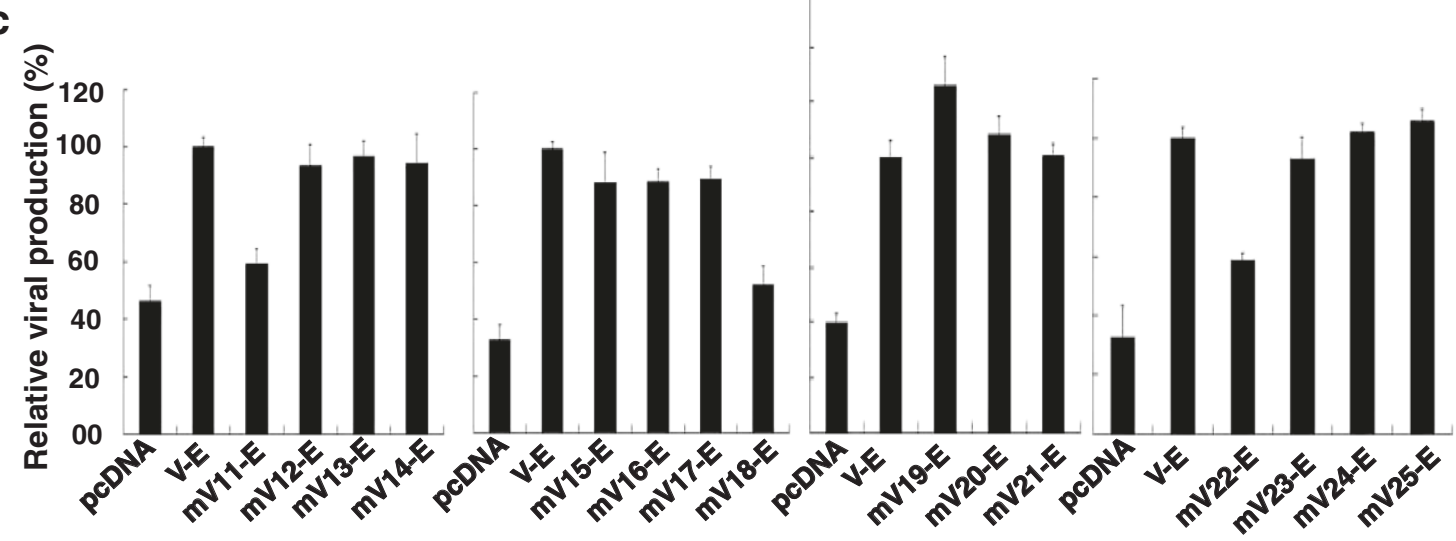

Figure $\mathbf{7}$ (See legend on next page.) 
(See figure on previous page.)

Figure 7 Functional validation of the crucial residues in the TMD of Vpu for BST-2 interaction. (a) Western blot analysis of the cellular lysates and the corresponding viral lysates following the cotransfection of the HIV-1 Gag-Pol construct (containing gag and pol genes) and other expressing plasmids into HEK293T cells. (b) Relative BST-2 reduction was determined by densitometric scanning using the ImageJ program (NIH). BST-2 level in cells that were cotransfected with HIV-1 Gag-Pol, R-B and pcDNA was arbitrarily set as 100\%. The data shown were the average of three independent experiments. (c) Relative HIV-1 VLPs release was assessed by densitometric scanning using the ImageJ program (NIH). The level of HIV-1 VLPs released from cells that were transfected with HIV-1 Gag-Pol, R-B and V-E was arbitrarily set as 100\%. The data shown were the average of three independent experiments.

HIV-1 Vpu TM that are important for their interaction. Mutations P40A in BST-2 and L11A, 14-16 (AII to VAA) and 26-28 (IIE to AAA) were first shown in our study to interfere with the interaction of Vpu and BST2. Results of molecular dynamics simulation further support this observation. Compared to the wild type complexes, the mutant complexes are less stable and show local conformation changes including the loss of helical structure and the formation of new helix. Such conformational changes possibly lead to the weakened interaction between BST-2 and Vpu. Further studies showed that the identified key residues in the TM domains of human BST-2 and HIV-1 Vpu were important for their respective functions. The loss of interaction between the two proteins directly affected the Vpumediated BST-2 degradation and the BST-2 inhibition of viral release.

BST-2/Vpu interaction has been used as the target to identify small molecules that antagonize HIV-1 Vpu function by cell-based ELISA monitoring the upregulation of cell surface BST-2 [37]. BRET assay is simple, rapid, non-invasive, and is suitable for developing a new high-throughput screening assay. The screening assay based on BRET method can lead to discovery of small molecules that disrupt the interaction between BST-2 and Vpu and rescue the antiviral activity of BST-2.

\section{Conclusions}

In this study, we utilized the BRET assay to further define the interaction of BST-2 and Vpu, and identified novel residues $\mathrm{P} 40$ in the TM domain of BST-2 and L11 in the TM domain of Vpu that were important for their interaction. Furthermore, triple-amino-acid substitutions, 14-16 (AII to VAA) and 26-28 (IIE to AAA) in Vpu TM, not single-residue mutation, exerted significant effect on BST2/Vpu interaction. The significant conformational changes of the BST2-Vpu complex structure caused by mutating P40 of BST-2 and L11, 14-16 (AII to VAA) and 26-28 (IIE to AAA) of $\mathrm{Vpu}$ were demonstrated by molecular dynamics (MD) simulation. In addition, disrupting interaction between the two proteins directly affected the subsequent BST2 degradation and the inhibition of viral release. These results add new insights into the molecular mechanism behind BST-2 antagonization by HIV-1 Vpu.

\section{Methods \\ Plasmids construction}

The human BST-2 (hBST-2) and HIV-1 Vpu cDNA were gifts from Chen Liang (Lady Davis Institute for Medical Research, McGill University). pR-B, pE-B, pB$\mathrm{R}, \mathrm{pB}-\mathrm{E}, \mathrm{pR}-\mathrm{Vm}, \mathrm{pE}-\mathrm{Vm}, \mathrm{pVm}-\mathrm{R}$ were generated by subcloning Renilla luciferase or EYFP cDNA and human BST-2 or Vpu cDNA into the pcDNA ${ }^{\mathrm{Tm}} 3.1 / \mathrm{V} 5-\mathrm{His}$ $A$ vector (Invitrogen). $\mathrm{pVm}-\mathrm{E}$ was generated by subcloning codon optimization Vm cDNA into pEYFP-N1 (Invitrogen). A series of BST-2 and Vpu TM mutants were generated by overlapping PCR-based mutagenesis. pE-CD209 was generated by subcloning EYFP cDNA and CD209 cDNA into the pcDNA $3.1 / \mathrm{V} 5$-His A vector (Invitrogen). All constructs were verified by nucleotide sequencing. The Vpu, hBST-2 and p24 antisera were obtained from the NIH AIDS Research and Reference Reagent Program. PEI (408727) was purchased from Sigma. Coelenterazine $\mathrm{h}$ was purchased from Promega.

\section{Cell culture and transfection}

Human embryonic kidney (HEK293T, ATCC \#CRL11268) cells were cultured in complete DMEM (Gibco) supplemented with $10 \%$ fetal calf serum (FCS), penicillin $(100 \mathrm{U} / \mathrm{ml})$, and streptomycin $(100 \mu \mathrm{g} / \mathrm{ml}) .8 \times 10^{5}$ cells/ well was seeded in 6-well plates one day prior to transfection. Cells were transfected with various plasmids depending on the experiment, using PEI (Sigma) according to the manufacturer's protocol.

\section{Immunofluorescence staining and confocal microscopy}

For immunostaining, cells on coverslips were transfected as described above and then fixed in $4 \%$ paraformaldehyde. After two washes in $1 \times$ phosphate buffered saline, the cells were permeabilized with $0.5 \%$ Triton $\mathrm{X}-100$. Staining was performed with primary antibodies including Rabbit BST-2 antiserum (1:1,000 dilution) and Vpu (1:1,000 dilution). Anti-rabbit FITC-conjugated or TRITC-conjugated antibodies (1:500 dilution) were used as secondary antibodies. Images were 
recorded with a Leica TCS SPE, DM2500 confocal microscope (Leica Microsystems).

\section{BRET assay}

HEK293T cells were seeded in six-well plates $\left(8 \times 10^{5}\right.$ cells per well) 24 hours prior to transfection. Plasmids were transfected using PEI (Sigma) according to the manufacturer's instructions. Forty-eight hours after transfection, cells were washed twice and detached into $1 \mathrm{ml} 1 \times$ phosphate buffered saline, and then distributed into 96-well microplates at $1 \times 10^{5}$ cells per well (Corning 3600 ). The substrate coelenterazine $\mathrm{h}$ (Promega) was added at a final concentration of $5 \mu \mathrm{M}$. Readings at $475 \mathrm{~nm}$ (reflecting the bioluminescence given off by Rluc) and $530 \mathrm{~nm}$ (reflecting the resonance energy transfer from Rluc to EYFP) were measured simultaneously by a Perkin Elmer instrument. The BRET ratio was calculated as emission at $530 \mathrm{~nm}$ (light emitted by EYFP)/emission at $475 \mathrm{~nm}$ (light emitted by Rluc). The BRET ratios reported were corrected by subtracting the ratios obtained in cells expressing the donor only (Rluc).

\section{Viral isolation}

To produce viruses, cells were seeded in 10-cm dishes $\left(5 \times 10^{6}\right.$ cells per dish) 24 hours prior to transfection. The HIV-1 Gag-Pol construct (containing gag and pol genes) and other plasmids were transfected using PEI (Sigma) according to the manufacturer's instructions. Forty-eight hours after transfection, the culture supernatants were filtered through a $0.45 \mu \mathrm{m}$ filter, and virus particles were pelleted by ultracentrifugation through a $20 \%$ sucrose cushion at $35,000 \mathrm{rpm}$ for 1 hour at $4^{\circ} \mathrm{C}$ using a Beckman SW 41Ti rotor.

\section{Western blotting}

Cells or viruses were lysed in RIPA buffer (0.1\% SDS, $1 \%$ Triton X-100, $1 \%$ sodium deoxycholate, $150 \mathrm{mM} \mathrm{NaCl}$, $10 \mathrm{mM}$ Tris [pH 7.5], $1 \mathrm{mM}$ EDTA), and equal amounts of cell or viral lysate were separated by SDS-PAGE on a $12 \%$ gel, followed by transferring proteins onto nitrocellulose membranes (Gelman Science). The membranes were probed with BST-2 antiserum (1:2,000 dilution), anti-Vpu serum (1:5,000 dilution), anti-p24 antibody (1:5,000 dilution), or a mouse monoclonal antibody against actin (1:5,000 dilution), followed by incubation with IRDye ${ }^{\mathrm{TM}}$ secondary antibodies $(1: 20,000)$. Protein bands were visualized with an LI-COR Odyssey instrument and quantified using the Image J automated digitizing program $(\mathrm{NIH})$.

Construction of initial model and MD simulations of BST2 TM and Vpu TM complex

The initial complex structures for BST-2 TMD peptide and Vpu TMD peptide were generated using Discovery
Studio 2.5 based the previous reported WT complex. The initial structures were embedded into an explicit DOPC lipid membrane including explicit solvent using Discovery Studio 2.5 to generate the input structures for the later molecular dynamic simulation in membrane. The membrane was generated using the VMD soft suit (25), following by the 3 ns pre-equilibrium in AMBER (University of California, San Francisco). The partial charges on DOPC monomer were calculated by fully reproducible ab-initio electronic structure computations with Gaussian 09 (Gaussian, Inc., Wallingford CT) using Hartree-Fock self consistent field (HF-SCF) with 6-31G* basis set after optimizing with DFT-B3LYP and successively with HF-SCF at 6-31G* level. The partial charges were then extracted following RESP protocol with ANTECHAMBER program of the AMBER suite. MD simulations were performed using the AMBER10 package, all-atom parm94 force fields, particle mesh-Ewald summation, SHAKE algorithm, and a time-step of $2 \mathrm{fs}$. The systems were minimized using the steepest decent method, followed by the conjugate gradient method to remove the bad contacts. An equilibrium period of 120 ps was performed before the MD simulations commenced. The $10 \mathrm{~ns}$ long MD simulations were con ducted in the NPT ensemble at $310 \mathrm{~K}$. After the simulation was completed, a cluster analysis was performed for the snapshots taken from the period of 3-9 ns, which amounts to 6000 snapshots for each simulation. Conformation clustering was performed with a radius of $2 \AA$ using the "Kclust" module implemented in the MMTSB software suite (The Scripps Research Institute). The obtained representative structure of the largest cluster family would be served as the representative conformation for the corresponding complex.

\section{Additional files}

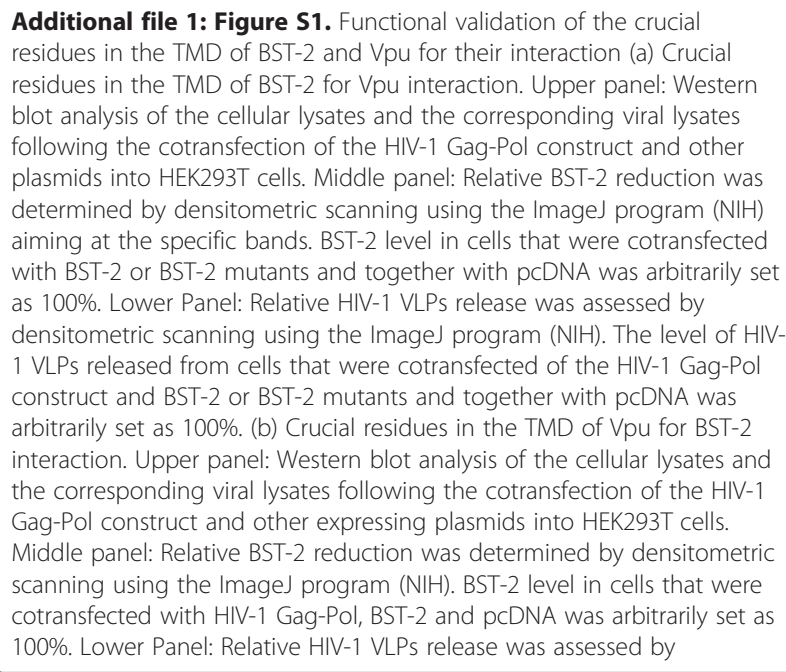


densitometric scanning using the ImageJ program (NIH). The level of HIV1 VLPs released from cells that were transfected with HIV-1 Gag-Pol and pcDNA was arbitrarily set as $100 \%$. In both middle and lower panels of (a) and (b), the data shown were the average of three independent experiments.

\section{Competing interests}

The authors declare that they have no competing interests.

\section{Authors' contributions}

FG, QJ, XP and SH conceived and designed the study. XP, SH, JL, FX, SM, and JZ carried out the experiments. SC, QJ and FG analyzed the data. FG, XP and SH drafted the manuscript. All authors read and approved the final manuscript.

\section{Acknowledgements}

We especially thank Chen Liang for providing various reagents and helpful comments and proofreading on the manuscript. This research was funded by grants from the Ministry of Science and Technology of China (2012CB911100, 2011CB504800, 2012ZX10001006, 2013ZX10001005 and 2010DFB30870) and from the Nature Science Foundation of China (30970155). The funding agencies had no role in the study design, data collection and analysis, the decision to publish, or preparation of the manuscript.

\section{Author details}

${ }^{1} \mathrm{MOH}$ Laboratory of Systems Biology of Pathogens, Institute of Pathogen Biology, Beijing 100730, P. R. China. ${ }^{2}$ Institute of Medicinal Biotechnology, Chinese Academy of Medical Sciences \& Peking Union Medical College, Beijing 100730, P. R. China.

Received: 25 March 2013 Accepted: 2 August 2013

Published: 6 August 2013

\section{References}

1. Van Damme N, Goff D, Katsura C, Jorgenson RL, Mitchell R, Johnson MC, Stephens EB, Guatelli J: The interferon-induced protein BST-2 restricts HIV-1 release and is downregulated from the cell surface by the viral Vpu protein. Cell Host Microbe 2008, 3:245-252.

2. Neil SJ, Zang T, Bieniasz PD: Tetherin inhibits retrovirus release and is antagonized by HIV-1 Vpu. Nature 2008, 451:425-430.

3. Kupzig S, Korolchuk V, Rollason R, Sugden A, Wilde A, Banting G: Bst-2/ HM1.24 is a raft-associated apical membrane protein with an unusual topology. Traffic 2003, 4:694-709.

4. Arias JF, Iwabu Y, Tokunaga $\mathrm{K}$ : Structural basis for the antiviral activity of BST-2/tetherin and its viral antagonism. Front Microbio/ 2011, 2:250.

5. Fitzpatrick K, Skasko M, Deerinck TJ, Crum J, Ellisman MH, Guatelli J: Direct restriction of virus release and incorporation of the interferon-induced protein BST-2 into HIV-1 particles. PLOS Pathog 2010, 6:e1000701

6. Hammonds J, Wang JJ, Yi H, Spearman P: Immunoelectron microscopic evidence for Tetherin/BST2 as the physical bridge between HIV-1 virions and the plasma membrane. PLoS Pathog 2010, 6:e1000749.

7. Perez-Caballero D, Zang T, Ebrahimi A, McNatt MW, Gregory DA, Johnson MC, Bieniasz PD: Tetherin inhibits HIV-1 release by directly tethering virions to cells. Cell 2009, 139:499-511.

8. Zhang J, Liang C: BST-2 diminishes HIV-1 infectivity. J Virol 2010, 84:12336-12343

9. Xu F, Tan J, Liu R, Xu D, Li Y, Geng Y, Liang C, Qiao W: Tetherin inhibits prototypic foamy virus release. Virol J 2011, 8:198.

10. Arnaud F, Black SG, Murphy L, Griffiths DJ, Neil SJ, Spencer TE, Palmarini M: Interplay between ovine bone marrow stromal cell antigen 2/tetherin and endogenous retroviruses. J Virol 2010, 84:4415-4425.

11. Hu S, Pang X, Li J, Cen S, Jin Q, Guo F: The role of the structural domains of human BST-2 in inhibiting the release of xenotropic murine leukemia virus-related virus. Biochem Biophys Res Commun 2012, 428:17-23.

12. Jones PH, Mehta HV, Maric M, Roller RJ, Okeoma CM: Bone marrow stromal cell antigen 2 (BST-2) restricts mouse mammary tumor virus (MMTV) replication in vivo. Retrovirology 2012, 9:10.
13. Jouvenet $N$, Neil SJ, Zhadina M, Zang T, Kratovac Z, Lee Y, McNatt M, Hatziioannou T, Bieniasz PD: Broad-spectrum inhibition of retroviral and filoviral particle release by tetherin. J Virol 2009, 83:1837-1844.

14. Strebel K, Klimkait T, Martin MA: A novel gene of HIV-1, vpu, and its 16-kilodalton product. Science 1988, 241:1221-1223.

15. Mangeat B, Gers-Huber G, Lehmann M, Zufferey M, Luban J, Piguet V: HIV-1 Vpu neutralizes the antiviral factor Tetherin/BST-2 by binding it and directing its beta-TrCP2-dependent degradation. PLoS Pathog 2009, 5:e1000574.

16. Skasko M, Wang Y, Tian Y, Tokarev A, Munguia J, Ruiz A, Stephens EB, Opella SJ, Guatelli J: HIV-1 Vpu protein antagonizes innate restriction factor BST-2 via lipid-embedded helix-helix interactions. J Biol Chem 2012, 287:58-67.

17. Douglas JL, Viswanathan K, McCarroll MN, Gustin JK, Fruh K, Moses AV: Vpu directs the degradation of the human immunodeficiency virus restriction factor BST-2/Tetherin via a \{beta\}TrCP-dependent mechanism. J Virol 2009, 83:7931-7947.

18. Mitchell RS, Katsura C, Skasko MA, Fitzpatrick K, Lau D, Ruiz A, Stephens EB, Margottin-Goguet F, Benarous R, Guatelli JC: Vpu antagonizes BST-2 -mediated restriction of HIV-1 release via beta-TrCP and endo-lysosomal trafficking. PLoS Pathog 2009, 5:e1000450.

19. Gupta RK, Hue S, Schaller T, Verschoor E, Pillay D, Towers GJ: Mutation of a single residue renders human tetherin resistant to HIV-1 Vpu-mediated depletion. PLoS Pathog 2009, 5:e1000443.

20. McNatt MW, Zang T, Hatziioannou T, Bartlett M, Fofana IB, Johnson WE, Neil SJ, Bieniasz PD: Species-specific activity of HIV-1 Vpu and positive selection of tetherin transmembrane domain variants. PLOS Pathog 2009, 5:e1000300.

21. Rong L, Zhang J, Lu J, Pan Q, Lorgeoux RP, Aloysius C, Guo F, Liu SL, Wainberg MA, Liang C: The transmembrane domain of BST-2 determines its sensitivity to down-modulation by human immunodeficiency virus type 1 Vpu. J Virol 2009, 83:7536-7546.

22. Guo F, Liang C: Transmembrane interactions of HIV-1 Vpu and tetherin. Curr HIV Res 2012, 10:292-297.

23. Tokarev A, Skasko M, Fitzpatrick K, Guatelli J: Antiviral activity of the interferon-induced cellular protein BST-2/tetherin. AIDS Res Hum Retroviruses 2009, 25:1197-1210.

24. Kobayashi T, Ode H, Yoshida T, Sato K, Gee P, Yamamoto SP, Ebina H, Strebel K, Sato H, Koyanagi Y: Identification of amino acids in the human tetherin transmembrane domain responsible for HIV-1 Vpu interaction and susceptibility. J Virol 2011, 85:932-945.

25. Vigan R, Neil SJ: Determinants of tetherin antagonism in the transmembrane domain of the human immunodeficiency virus type 1 Vpu protein. J Virol 2010, 84:12958-12970.

26. Pfleger KD, Seeber RM, Eidne KA: Bioluminescence resonance energy transfer (BRET) for the real-time detection of protein-protein interactions. Nat Protoc 2006, 1:337-345.

27. Pfleger KD, Eidne KA: Illuminating insights into protein-protein interactions using bioluminescence resonance energy transfer (BRET). Nat Methods 2006, 3:165-174

28. $\mathrm{Xu}$ Y, Piston DW, Johnson $\mathrm{CH}$ : A bioluminescence resonance energy transfer (BRET) system: application to interacting circadian clock proteins. Proc Natl Acad Sci USA 1999, 96:151-156.

29. Subramanian C, Kim BH, Lyssenko NN, Xu X, Johnson CH, von Arnim AG: The Arabidopsis repressor of light signaling, COP1, is regulated by nuclear exclusion: mutational analysis by bioluminescence resonance energy transfer. Proc Natl Acad Sci U S A 2004, 101:6798-6802.

30. Kroeger KM, Eidne KA: Study of G-protein-coupled receptor-protein interactions by bioluminescence resonance energy transfer. Methods $\mathrm{Mol}$ Biol 2004, 259:323-333.

31. Kuhl BD, Cheng V, Donahue DA, Sloan RD, Liang C, Wilkinson J, Wainberg MA: The HIV-1 Vpu viroporin inhibitor BIT225 does not affect Vpumediated tetherin antagonism. PLoS One 2011, 6:e27660.

32. Zhou J, Zhang Z, Mi Z, Wang X, Zhang Q, Li X, Liang C, Cen S: Characterization of the interface of the bone marrow stromal cell antigen 2-Vpu protein complex via computational chemistry. Biochemistry 2012, 51:1288-1296.

33. Banning C, Votteler J, Hoffmann D, Koppensteiner $H$, Warmer M, Reimer $R$ Kirchhoff F, Schubert U, Hauber J, Schindler M: A flow cytometry-based FRET assay to identify and analyse protein-protein interactions in living cells. PLoS One 2010, 5:e9344. 
34. McNatt MW, Zang T, Bieniasz PD: Vpu binds directly to tetherin and displaces it from nascent virions. PLoS Pathog 2013, 9:e1003299.

35. Skasko M, Tokarev A, Chen CC, Fischer WB, Pillai SK, Guatelli J: BST-2 is rapidly down-regulated from the cell surface by the HIV-1 protein Vpu: evidence for a post-ER mechanism of Vpu-action. Virology 2011, 411:65-77.

36. Lv M, Wang J, Wang X, Zuo T, Zhu Y, Kong W, Yu X: Polarity changes in the transmembrane domain core of HIV-1 Vpu inhibits its anti-tetherin activity. PLoS One 2011, 6:e20890.

37. Zhang Q, Liu Z, Mi Z, Li X, Jia P, Zhou J, Yin X, You X, Yu L, Guo F, et al: High-throughput assay to identify inhibitors of Vpu-mediated downregulation of cell surface BST-2. Antiviral Res 2011, 91:321-329.

doi:10.1186/1742-4690-10-84

Cite this article as: Pang et al:: Identification of novel key amino acids at the interface of the transmembrane domains of human BST-2 and HIV-1 Vpu. Retrovirology 2013 10:84.

\section{Submit your next manuscript to BioMed Central and take full advantage of:}

- Convenient online submission

- Thorough peer review

- No space constraints or color figure charges

- Immediate publication on acceptance

- Inclusion in PubMed, CAS, Scopus and Google Scholar

- Research which is freely available for redistribution 